\title{
Application of Passivation Phenomena to Chemical Sensors I. The Simultaneous Monitoring of the High Concentrations of Hydrogen Ion and Sulfate Ion
}

\author{
Akimitsu ISHIHARA* and Shukuji ASAKURA
}

Reccived July 11, 1990 ; Accepted September 12, 1990

\begin{abstract}
'The authors proposed a new method measuring hydrogen ion and sulfate ion in concentrated acids by using passivation and depassivation phenomena. The basic idea underlying this method is that the chemical composition of a solution sensitively reflect on the transitions between active and passive states. So far, very few antecedent studies have been established on this principle.

In the present study, a new method using passivation and depassivation phenomena has successfully determined the concentrations of hydrogen ion and sulfate ion separately. Both passivation and depassivation time are the functions of the concentrations of hydrogen ion and sulfate ion. The contour lines of passivation and depassivation $t$ ime are plotted on a single plane. The intersecting point of these two lines gives the concentrations of hydrogen ion and sulfate ion ion the test solution.
\end{abstract}

\section{I . INTRODUCT ION}

The continuous and sensitive monitoring of concentrated acids in chemical industries is indispensable. However, the conventional chemical sensors, e.8. pH glass electrode, do not operate properly in these media because of the deterioration and pollution of sensing materials" . There have been very few sensors satisfying the industrial requirements in these severe envi ronments.

In this paper, the authors propose a new method sensing the high concentrations of hydrogen ion and sulfate ion simultaneously. The sensitive dependence of anodic

Department of Materials Fingineering, Faculty of Engineering, Yokohama National University

I56 Tokiwadai Hodogaya-ku Yokohama-shi Kanagawa-ken

Key word: Passivation, Chemical sensor, Industrial measurement, Concentrated acid solution passivation of iron on a chemical enviromment is utilized. The present method is proved to be free from the pollution of electrode surface giving the reproducible output.

\section{EXPER I HENTAI}

A commercial grade iron, SM4l steel, was used for the sensing electrode. The iron rod with a $8 \mathrm{~mm}$ diameter embeded in teflon holder was installed to a rotating disk device so as the electrode surface was oriented downwards. The surface of iron electrode was polished with emery paper up to No.1000 and degreased with ethyl alcohol. The iron was anodically polarized at a constant current in sulfuric acid solutions with or without potassium sulfate. The cell for the measurement was $400 \mathrm{ml}$ glass vessel in which platinum counter electrode and a salt bridge were placed. The solution in the measuring vessel was connected to SCE through double salt bridges as shown in Fig.1. The solution 
was kept. at $25^{\circ} \mathrm{C}$ and was not deaerated.

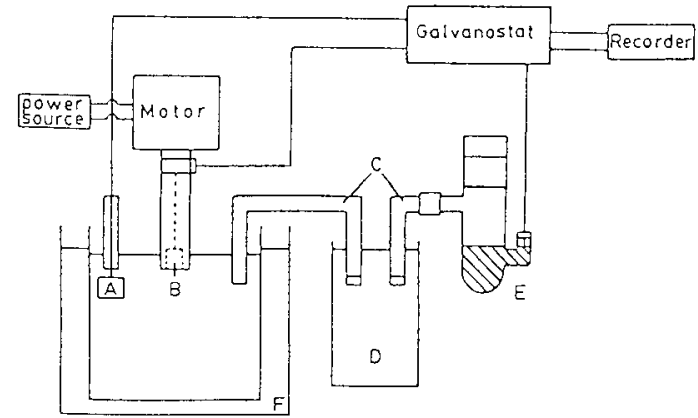

Fig.1. Apparatus $A$ : counter electrode $B$ :working electrode C:salt bridge D:saturated $\mathrm{K}_{2} \mathrm{SO}_{4} \mathrm{E}$ : Saturated Calomel Electrode.

The passivation and depassivation phenomena were observed according to the following processes. Figure 2 illustrates the procedure of experiment and responding potential change.

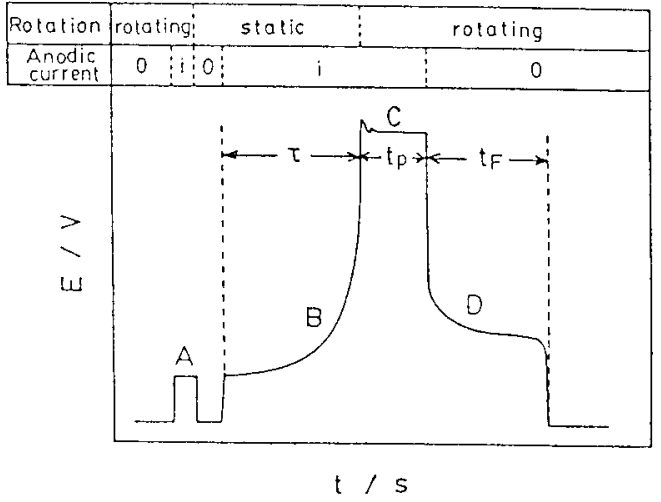

Fig.2. Potential-time curve for galvanostatic passivation and spontaneous decay of passivity as a function of time.

$t=$ Passivation time, $t_{F}=F l a d e t i m e$

\section{(1) Surface Cleaning (Process A)}

The electrode was conditioned by applying anodic current $i$ for 8 seconds under a rotating condition at speed $r$. In the current range of the present experiment, slight potential transient was observed, indicating the anodic dissolution of iron in an active state.

Then, the current was cut for a while in keeping the electrode rotating to remove the reaction product from electrode surface.

(2) Passivation (Process B)
Ihe rotation of electrode was stopped to prepare a static condition in the solution. Then, a constant anodic current $i$ having the same value as in Process $A$ was imposed. The attainment to passive state was monitored by an abrupt potential increase. The time requires to passive state was defined as passivation time $\tau$.

(3) Stabilization of Passive state (Process $C$ )

To stabilize the passive state, the current i was kept flowing for a period $t_{p}$. The electrode rotated in this process and the next at speed $r$ whose value was same as in Process $A$.

(4) Arrest at Flade Potential (Process D)

The arrest of potential which was socalled as Flade potential was observed after the current was shut of ${ }^{2}$. The $t$ ime $t_{F}$ staying at Flade potential was measured as illustrated in Fig.2. Figure 3 is a typical example of Fig. 2 .

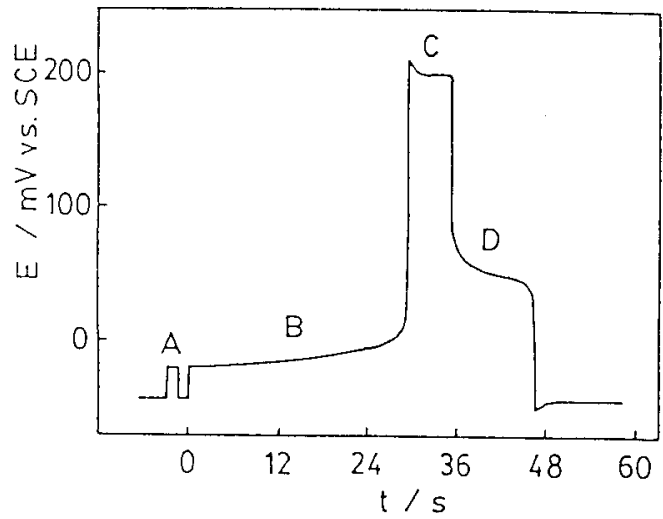

Fig.3. An actual example of Fig.2. $\left[\mathrm{H}_{2} \mathrm{SO}_{4}\right]=2.5 \mathrm{~mol} \cdot \mathrm{I}^{-1},\left[\mathrm{~K}_{2} \mathrm{SO}_{1}\right]=0.5 \mathrm{~mol} \cdot 1.1$ $\mathrm{i}=300 \mathrm{~mA} \cdot \mathrm{cm}^{-2}$.

\section{RESULTS AND DISCUSSION}

The preliminary experiments guaranteed that the highly reproducible data were obtained after the above processes were repeated a few times. Thus, all data presented in this paper were picked up after the reproducibility of data was confirmed by repeating the above processes. The practical discussion on reproducibility is given later in this paper. 
The effect of rotation speed $r$ on passivation time is shown in Fig.4.

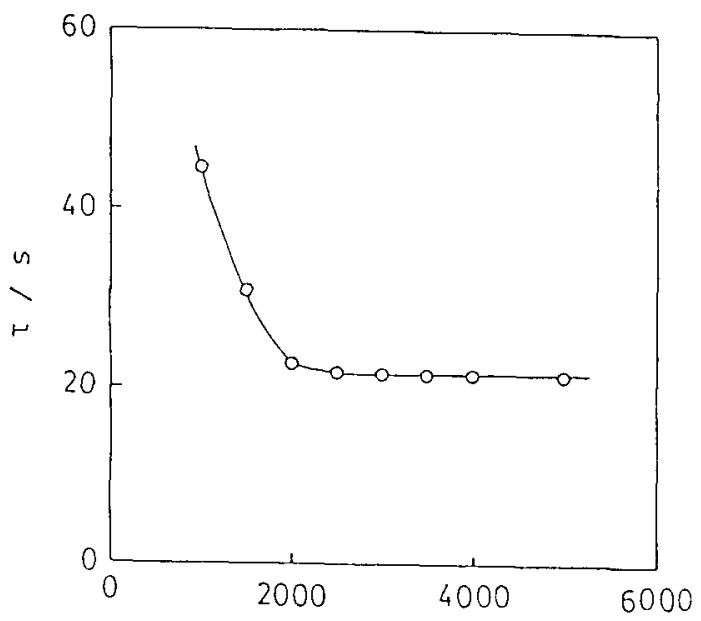

Rotation speed / rpm

Fig.4. Effect of the rotation speed on passivation time in $2 M$ sulfuric acid containing $1 M$ potassium sulfate.

This figure indicates that $\tau$ reaches a constant value for $r>2000 \mathrm{rpm}$. This means that the dissolved materials from the surface in Process A are swept away almost completely where $r$ exceeds 2000 rpm. The excellent reproducibility of potential increases were obtained for $r>2000 \mathrm{rpm}$. In contrast, some reaction products $^{31}$ in Process A seemed to remain when $r$ was less than 2000 rpm. Therefore, in all of the following experiments, rotation speed was adjusted to $2500 \mathrm{rpm}$.

First, passivation time $\tau$ is discussed. The logarithm of passivation time $\tau$ is plotted against logarithm of applied anodic current density $i$ in Fig. 5 .

Linear relations were obtained in all solutions of sulfuric acid with and wi thout potassium sulfate. Frank" found the relation in $0.5 \mathrm{~mol} / \mathrm{l}$ sulfuric acid

$$
\tau=\mathrm{k}\left(\mathrm{i}-\mathrm{i}_{0}\right)^{-2}
$$

where $i_{0}$ is a minimum current density required for passivation, $k$ is constant. In the present experiment,

$$
\tau=\mathrm{k} \cdot \mathrm{i}^{-8}\left(\tau: \mathrm{sec}, \mathrm{i}: \mathrm{mA} \cdot \mathrm{cm}^{-2}\right)
$$

was obtained. The sensitive dependence of $\tau$ on $i$ appears in Equation (2).

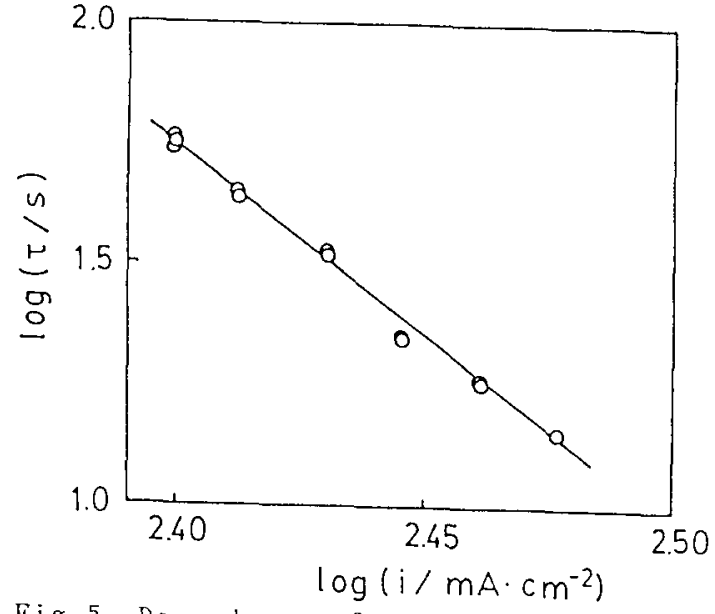

Fig.5. Dependence of passivation time on anodic current density of iron in $3 M$ sulfuric acid.

Passivation time was also affected by the concentrations of sulfuric acid and potassium sulfate to a great extent. $\tau$ was measured in the different sulfuric acid solutions being shown in Fig.6.

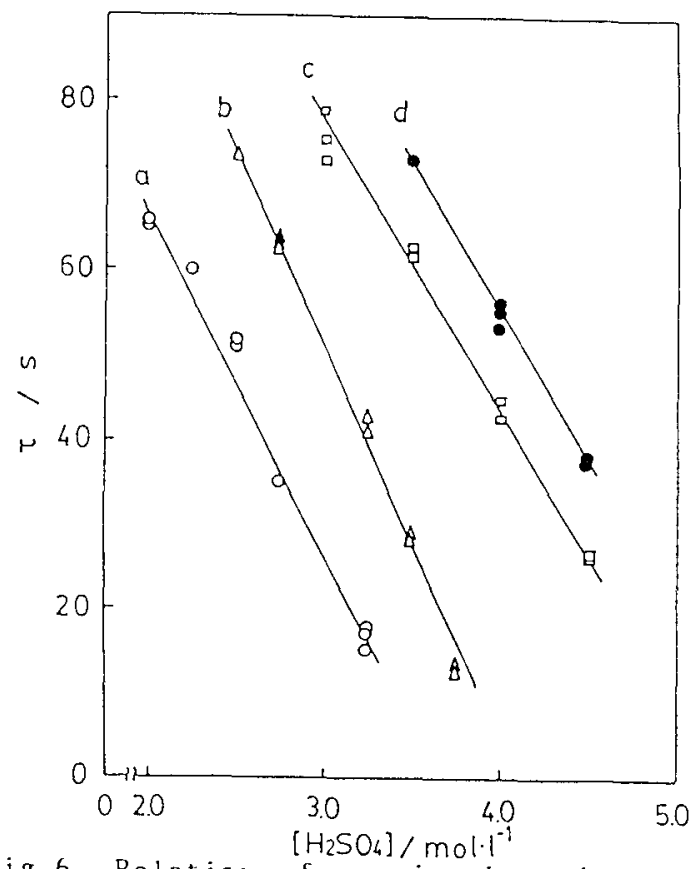

Fig.6. Relation of passivation time to the concentration of sulfuric acid.
(a) $360 \mathrm{~mA} \cdot \mathrm{cm}^{-2}$
(b) $300 \mathrm{~mA} \cdot \mathrm{cm}^{-2}$
(c) $260 \mathrm{~mA} \cdot \mathrm{cm}^{-2}$
(d) $250 \mathrm{~mA} \cdot \mathrm{cm}^{-2}$

This figure suggests the possibility of extremely sensitive and accurate 
determination of sulfuric acid concentration in narrow range between 2 mol $1^{-1}$ and 5 thanks to the steep dependence of $\tau$ on the concentration. The high reproducibility was not obtained below I mol-1 l sulfuric acid. Ihe complicated dependence of $\tau$ on the composition of the solution appeared where the sulfuric acid concentration exceeded 5 mol $\cdot 1^{-1}$. Therefore, the concentration range of sulfuric acid measurable by the present method is 2 mol $\cdot 1^{-1}$ to 5 .

Passivation time was also measured in the solution containing sulfuric acid and potassium sulfate. The effect of sulfate ion is given in Fig.7.

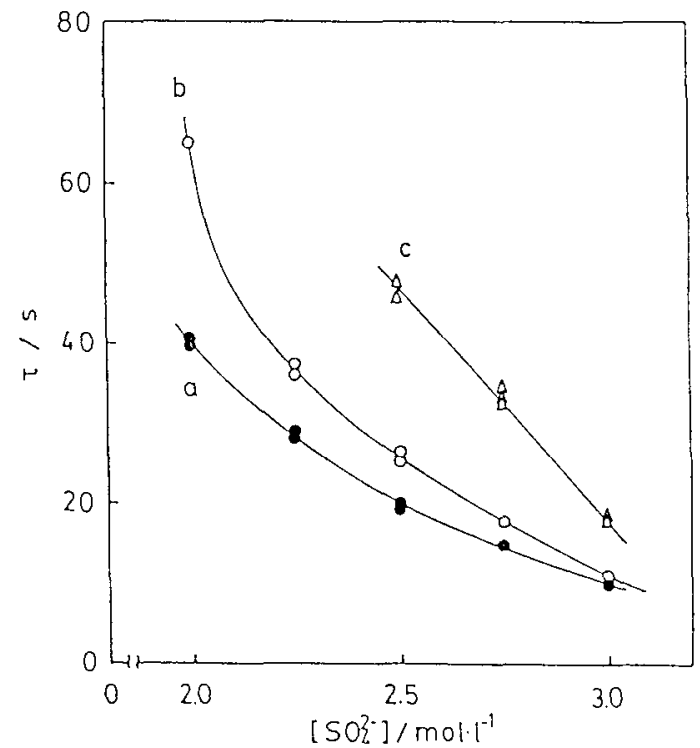

Fig.7. Plot of passivation time against the total concentration of sulfate ion in $2 \mathrm{M}$ sulfuric acid.

(a) $380 \mathrm{~mA} \cdot \mathrm{cm}^{-2}$

(b) $360 \mathrm{~mA} \cdot \mathrm{cm}^{-2}$

(c) $300 \mathrm{~mA} \cdot \mathrm{cm}^{-2}$

Figures 6 and 7 indicate that passivation time is a function of the concentrations of hydrogen ion and sulfate ion, and anodic current density. By introducing empirical function $f_{1}$, the relation is expressed by

$$
\tau=\mathrm{f}_{1}\left(\left[\mathrm{H}^{+}\right],\left[\mathrm{SO}_{4}{ }^{2-}\right], \mathrm{i}\right)
$$

where $\left[\mathrm{H}^{+}\right]$and $\left\{\mathrm{SO}_{4}{ }^{2} \mid\right.$ are the concentrations of hydrogen ion and sulfate ion, respectively. Since anodic current density can be maintained constant, $\tau$ becomes the function of the concentrations of these ions. Equation (3) provides the contour lines of passivation time between $\left[H^{+}\right]$and [ $\left.\mathrm{SO}_{4}{ }^{2-}\right]$. A few contour lines of passivation time are drawn in Fig.8.

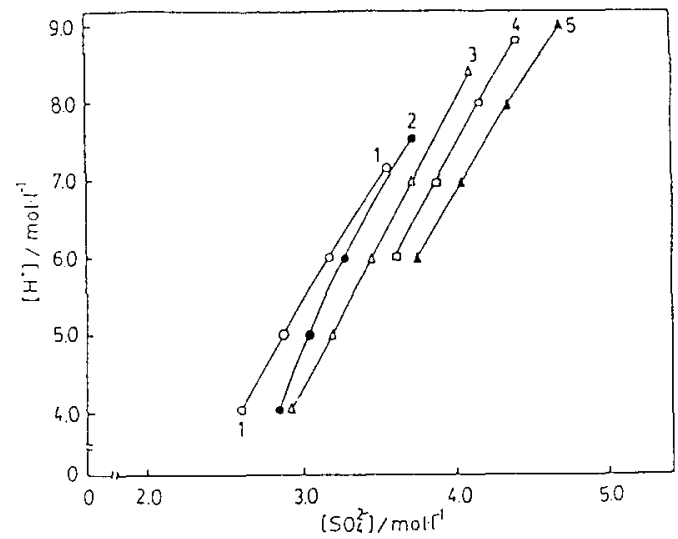

Fig.8. Contour lines of passivation time.

$260 \mathrm{~mA} \cdot \mathrm{cm}^{-2}$ (1) $\tau=60 \mathrm{sec}$ (2) $50 \mathrm{sec}$ $250 \mathrm{~mA} \cdot \mathrm{cm}^{-2}$ (3) $50 \mathrm{sec}$ (4) $40 \mathrm{sec}$

(5) $20 \mathrm{sec}$

In this figure, the combinations of $\left[\mathrm{H}^{+}\right]$and $\left[\mathrm{SO}_{4}{ }^{2-}\right]$ or a contour 1 ine give the equal passivation time. Hence, if a concentration of either hydrogen ion or sulfate ion is known or measured by a different method, the other can be determined from passivation time.

Next, the interval $t_{F}$ where the iron maintains passive state after an anodic current is shut of $\mathrm{is}$ discussed. The transition of passive state to active one was monitored from the sudden decay of Flade potential as shown in Fig.2. Actually, time $t_{F}$ is the interval where Flade potential appears. In this aspect, $t_{F}$ is named as Flade time in this paper. Flade time was found not to be dependent on the anodic current density $i$ and the time $t_{p}$ of process $C$, as observed in Figs. 9 and 10 .

This surmises that the passive layer once formed does not grow rapidly. In practical sense, once the passive state is accomplished, the depassivation process is independent of processes A-B$C$ in Fig.2. Flade $t$ ime $t_{F}$ measured in sulfuric acid is given in Fig. 11 . 


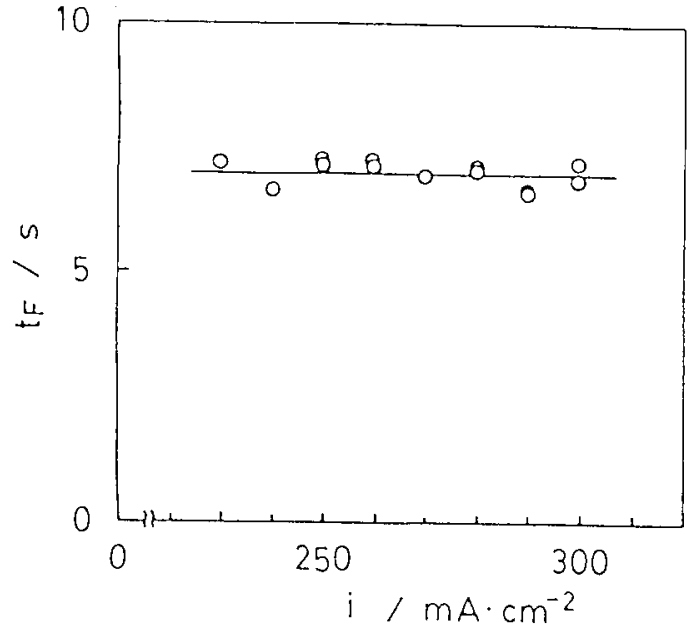

Fig.9. Indifference of Flade time to anodic current density in $4 M$ surfuric acid.

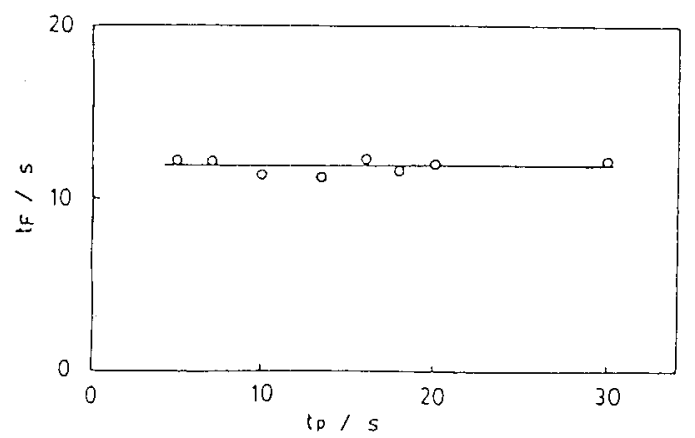

Fig.10. Dependence of Flade time on the time of process $C t_{p}$ in $2 M$ sulfuric acid containing 0.3M potassium sulfate.

Effect of sulfate ion on Flade time $t_{F}$ in sulfuric acid was also studied by adding potassium sulfate as shown in Fig. 12 .

Figures 11 and 12 indicate Flade time $t_{r}$ is the function of the concentrations of hydrogen ion and sulfate ion. Flade time $t_{F}$ is written mathematically as

$$
t_{F}=f_{2}\left(\left[H^{*}\right],\left[\mathrm{SO}_{4}{ }^{2-}\right]\right)
$$

where $f_{2}$ means a empirical function. The contour lines of $t_{r}$ between the concentrations of hydrogen ion and sulfate ion can be drawn in the same manner as Fig.8. A few contour 1 ines for different $t_{F}$ are plotted in Fig.13.

If the concentration of hydrogen ion is known, that of sulfate ion can be determined from flade time $t r$, vice versa.

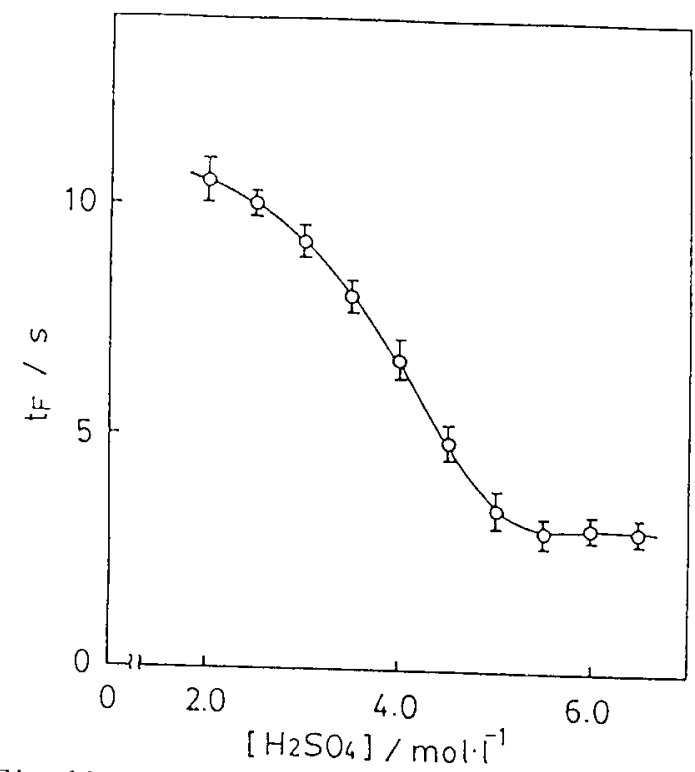

Fig.11. Effect of the concentration of sulfuric acid on Flade time.

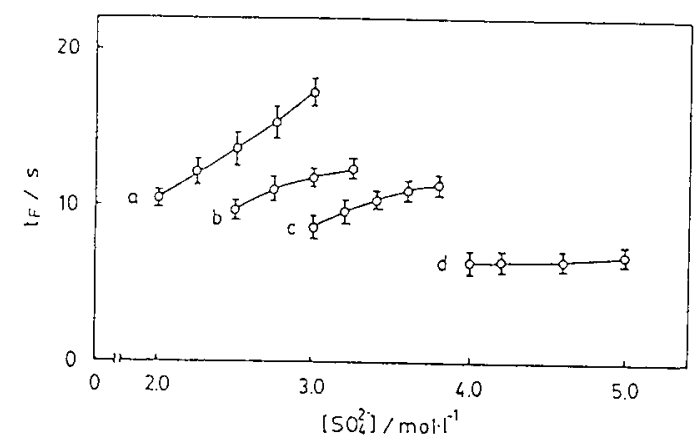

Fig.12. Plot of Flade time against the total concentration of sulfate ion.
(a) $\left[\mathrm{H}^{+}\right]=4.0 \mathrm{M}$
(d) $\left[\mathrm{H}^{+}\right]=8.0 \mathrm{M}$
$\begin{array}{ll}\text { (b) }\left[\mathrm{H}^{+}\right]=5.0 \mathrm{M} & \text { (c) }\left[\mathrm{H}^{+}\right]=6.0 \mathrm{M}\end{array}$

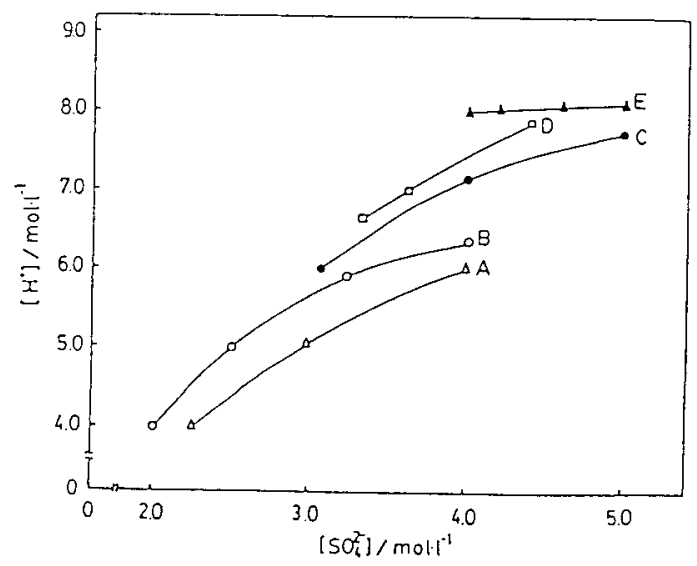

Fig. 13. Contour lines of Flade time.

(A) $t_{r}=12 \mathrm{sec}$ (B) $10 \mathrm{sec}$ (C) $9 \mathrm{sec}$

(D) $8 \mathrm{sec}$ (E) $6.4 \mathrm{sec}$ 
The analyses of passivation time $\tau$ and Flade time tr having been made in this paper manifests that both values are the functions of the concentrations of hydrogen ion and sulfate ion. The combination of two contour lines appearing in Fig.8 and 13 realizes the simultaneous determination of the concentrations of hydrogen ion and sulfate ion. The derivation process of these two concentrations is explained in Fi g. 14 .

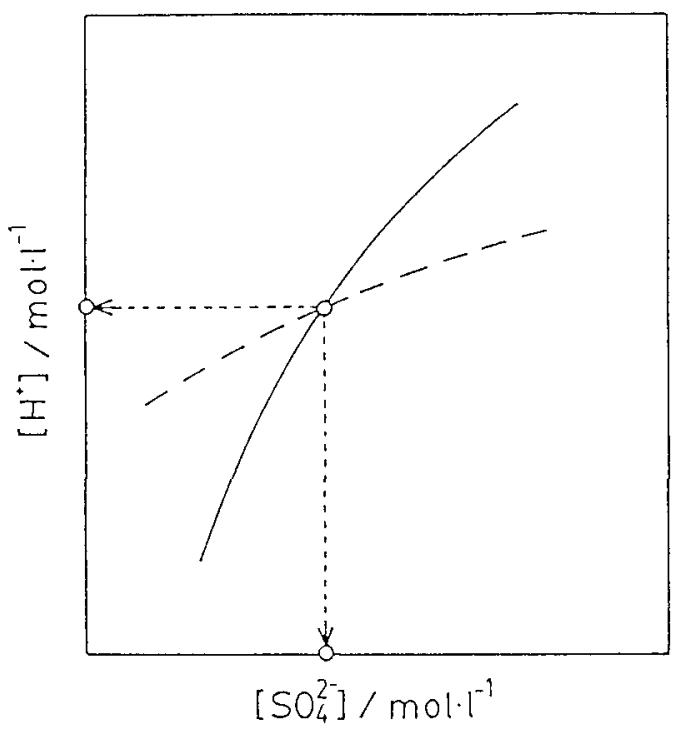

Fig.14. Illustration for the determination of the concentrations of hydrogen ion and sulfate ion.

- : contour line of passivation time - - contour line of Flade time

First, passivation time $\tau$ and Flade time $t_{r}$ are determined in a test solution containing hydrogen ion and sulfate ion.

Second, the contour lines of passivation time $\tau$ and Flade time $t_{r}$ corresponding to the measured values are found in Fig.8 and 13 .

Third, these two contour 1 ines are plotled a single plane as shown in Fig. 14. The intersecting point of these two 1 ines gives the concentrations of hydrogen ion and sulfate ion in the test solution. These two contour lines are actually plotted in Fig. 15 .

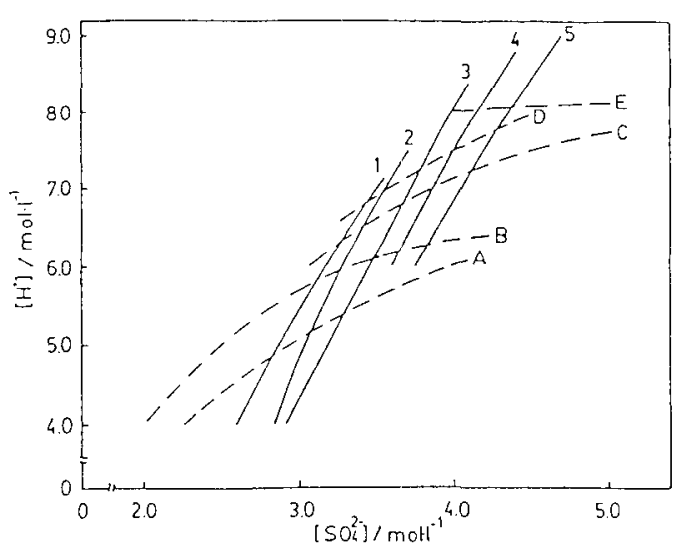

Fig.15. Contour lines of passivation time and Flade time.

Passivation time

$\begin{aligned} 260 \mathrm{~mA} \cdot \mathrm{cm}^{-2} & \text { (1) } t=60 \mathrm{sec}(2) 50 \mathrm{sec} \\ 250 \mathrm{~mA} \cdot \mathrm{cm}^{-2} & \text { (3) } 50 \mathrm{sec}(4) 40 \mathrm{sec} \\ & \text { (5) } 20 \mathrm{sec}\end{aligned}$

Flade time (A) $t_{F}=12 \mathrm{sec}$ (B) $10 \mathrm{sec}$

(C) $9 \mathrm{sec}$ (D) $8 \mathrm{sec}$ (E) $6.4 \mathrm{sec}$

Though the conceutration ranges are not wide, the resolution of the measurand (quantity to be measured) is very high. finally, the reproducibility of results in the present method should be discussed. Passivation time $\tau$ and Flade time $t_{F}$ were highly reproducible as long as the measuring process given in Fig.2 was followed. A proof is given in Fig. 16, in which passivation time $\tau$ becomes constant after the measuring processes were repeated 4 to 5 times.

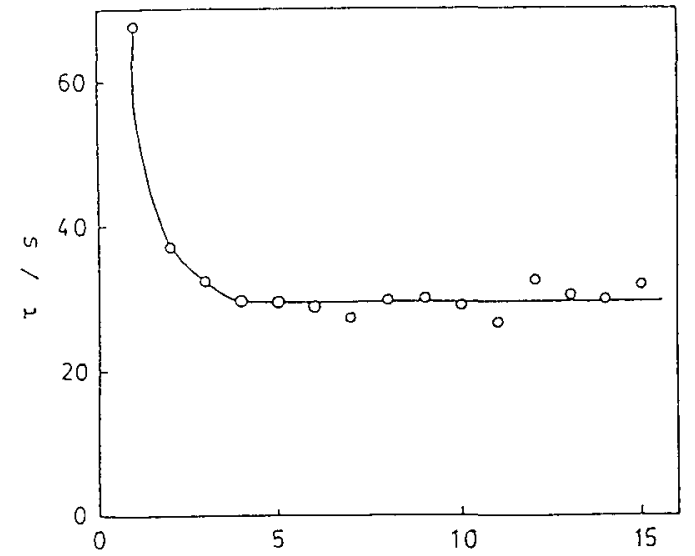

number of repititions of measurement

Fis.16. reproducibility of passivation time in $2.5 \mathrm{M}$ sulfuric acid. $i=300 \mathrm{~m} \Lambda \cdot \mathrm{cm}^{-2}$ 
The high reproducibility could result from the continuous surface cleaning of the electrode due to corrosion and anodic dissolution. Since the sensing electrode is comsumptive in the present method, no maintenance for continuous use might be required.

\section{CONCL.US ION}

The authors succeeded in determining the high concentrations of hydrogen ion and sulfate ion simultaneously by using passivation and depassivation phenomena of iron. The advantages of the present method are as follows.

(1) The maintenance of sensing electrode is not always needed because of the continuous renewal of the surface due to dissolution.

(2) Since the present method requires only the transient response of electrode potential, there is no need measuring the electrode potential exactly.

(3) As the impedance of measuring system is low, the environmental noise can be minimized.

The expansion of the sensing material to other metals and alloys than iron would provide versatile possibilities of sensing various chemical species.

The additional experiments showed that the effect of chloride and nitrate was not negligible. The studies on this effect are now going.

\section{REFERENCES}

1) I.Voge1."A Pextbook of Quantitative Inorganic Analysis including Elementary Analysis",4th Ed., Longman, New York, 1978 , Chapter XV.

2)F.Flade, 7.Phys.Chem.76,513(1911).

3)G.Gilli and F.Zucchi, Corr.Sci. 8,801 $(1968)$.

4) U.F.Frank, Z.Naturforsh.4a,378(1949). 